\title{
Endurance vs. strength exercise
}

\author{
Narayan Bahadur Mahotra
}

\section{Author(s) info:}

Department of Clinical Physiology, Maharajgunj Medical Campus, Institute of Medicine, Maharajgunj, Kathmandu, Nepal

\section{Correspondence:}

Dr. Narayan Bahadur Mahotra $M B B S, M D$

Email:

narayanmahotra@gmail.com

DOI :

https://doi.org/10.3126/jpsn.v1i2.42275

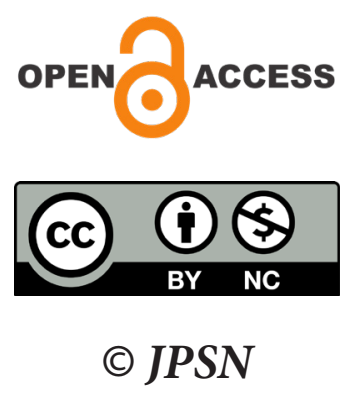

Endurance and strength are separate physical qualities of exercise. Both have great importance in the sporting performance as well as in the physical health.[1] Exercise that is of low intensity, low resistance but long duration such as running, cycling or swimming is called endurance exercise.[1,2] Endurance exercise helps increase the capacity of a muscle or a group of muscles to engage in physical activities for longer periods of time before fatigue sets in. Endurance exercise, also called aerobic exercise, increases the number of mitochondria in the muscle fibers. $[1,3,4]$ It also increases the number of capillaries around the muscle fibers. With endurance exercise, there is a decrease in the number of fast glycolytic muscle fibers and an increase in the number of fast oxidative muscle fibers as the oxidative capacity of muscle fibers increases. All these changes lead to an increase in the capacity for endurance activity with a minimum of fatigue. $[4,5,6]$

Endurance exercise brings changes not only in the skeletal muscles but also in the respiratory and cardiovascular systems thereby improving the delivery of oxygen and nutrients to the muscle as well as other body tissues. $[5,6]$ There are three primary factors that account for the endurance performance: maximum oxygen consumption ( $\left.\mathrm{Vo}_{2} \max \right)$, exercise economy and lactate threshold.[4] $\mathrm{Vo}_{2}$ max can be defined as the highest rate at which $\mathrm{O}_{2}$ can be transported and utilized by the body during maximal exercise.[4] It has long been considered one of the most important factors in determining endurance performance. Exercise economy is the $\mathrm{O}_{2}$ consumption required at a given absolute submaximal exercise intensity. [4] Lactate threshold can be defined as the fraction of the maximum $\mathrm{O}_{2}$ uptake at which blood lactate rapidly rises.[4] It has been hypothesized that the lactate threshold can be increased by an improvement in work efficiency, if the athlete's body mass and absolute $\mathrm{Vo}_{2}$ max remain constant. [4]

In contrast, high intensity, high resistance but short duration exercise such as weight lifting is called strength exercise.[1,4,5] It helps to build muscle mass, muscle tone and strength. In strength exercise, fast glycolytic muscle fibers are recruited during contractions. These muscle fibers undergo hypertrophy due to the increased synthesis of actin and myosin filaments of myofibrils.[5,6] In addition, glycolytic activity is also increased by increasing the synthesis of glycolytic enzymes. These muscles, although very powerful, have little capacity for endurance and get fatigue easily. $[5,6]$ These changes in muscle in response to repeated exercise occur slowly over a period of weeks. If regular exercise ceases, the changes in the muscle that occurred as a result of the exercise will slowly revert back to unexercised state. $[1,5,6]$ 
There is increase in skeletal muscle strength and cross-sectional area with strength exercise. These changes are primarily the product of neuromuscular alterations, skeletal muscle plasticity and metabolic improvements. Neuromuscular alterations include increase in rate of force development, motor unit synchronization, frequency modulation, autogenic inhibition and reduced antagonist inhibition. $[3,4,6]$ Skeletal muscle plasticity refers to increase in size of individual muscle fibers, muscle fiber type transitioning and structural alterations; metabolic improvements refer to increase in muscle glycogen stores and increase in enzymes associated with the ATP and phosphocreatine.[3,4,6] Other changes include bone mineral density and increase connective tissue stiffness. [3,4]

Though the ability of a muscle to adapt to exercise decreases with age, the regular exercise can produce significant adaptation even in the elderly. Endurance exercise is very important in elderly as it has a very good effect on the cardiovascular system. Similarly, modest degree strength exercise can partially prevent the loss of muscle tissue that occurs with aging. Moreover, it helps maintain stronger bones and joints. $[4,6]$

Strength exercise is seen as a means of increasing the level of sporting achievement. By developing this quality, the capabilities of the athletes in power sports are improved. Strength does not interfere with sports with predominant manifestation of endurance. Moreover, it has a positively stimulating influence on the endurance. $[1,4,5]$

So, both the strength and endurance exercise can be combined for the athletes. Different types of exercise produce different changes in the endurance and strength capacity of a muscle. Therefore, a person should choose a type of exercise compatible with the type of activity he/she ultimately wishes to perform. $[1,4,5]$

\section{REFERENCES}

1. Rohleva M. Exploring the relationship between endurance and strength training in power sports. Journal of Education and Training Studies. 2017; 4(5): 204-208.

2. Free from Falls. The National Multiple Sclerosis Society. 2020.

3. Williams J. Concurrent strength and endurance training. $J$ Phy Fit Treatment and Sports. 2017; 1(3):1-5

4. Lewis M. Strength training and endurance athletes. Strength and Conditioning. 2015; 2(4):1-8.

5. Hickson RC et al. Potential for strength and endurance training to amplify endurance performance. Journal of Applied Physiology. 1988; 2285-2290.

6. Widmaier P Eric, Raff Hershel, Strang T Kevin. Vander's human physiology - the mechanism of body function. Eleventh edition. Mc Graw Hill higher education; 2008. 